\title{
EFFECT OF NEUTRON IRRADIATION ON THE EPR SPECTRA OF $\mathrm{CuGeO}_{3}$
}

\section{E. Mosiniewicz-SzaBlewska ${ }^{a}$, L. GeadczuK ${ }^{a}$, H.A. DąBKowska ${ }^{b}$,} B. PYTEL ${ }^{c}$ AND H. SZYMCZAK ${ }^{a}$

${ }^{a}$ Institute of Physics, Polish Academy of Sciences Al. Lotników 32/46, 02-668 Warsaw, Poland

${ }^{b}$ Brockhouse Institute for Materials Research, McMaster University, Hamilton, Canada.

${ }^{c}$ Institute of Atomic Energy, Otwock-Świerk, Poland

The influence of neutron irradiation on the electron paramagnetic resonance spectra of the spin-Peierls compound $\mathrm{CuGeO}_{3}$ was investigated in the wide temperature range $2-300 \mathrm{~K}$. It was found that the irradiation induced appreciable changes in the EPR signal intensity, resonance line width and $g$-factor of this material. It was also shown that the irradiation leads to a decrease in the spin-Peierls transition temperature. For higher irradiation doses, an antiferromagnetically ordered phase can be observed at lower temperatures. This phase coexists with the spin-Peierls phase. The observed decrease in the spin-Peierls transition temperature after irradiation may be associated with some changes in topological and chemical short-range order or with the transmutation of the fraction of ${ }^{63} \mathrm{Cu}$ in the ${ }^{63} \mathrm{Cu}(\mathrm{n}, \alpha){ }^{60} \mathrm{Co}$ reaction.

PACS numbers: $75.30 . \mathrm{Kz}, 75.50 . \mathrm{Ee}, 76.30 . \mathrm{Da}$

\section{Introduction}

The compound $\mathrm{CuGeO}_{3}$ is presently a subject of high interest $[1,2]$ because it presents a transition into a spin-Peierls (SP) ground state at low temperatures. The unusual properties of this compound are the consequence of a pronounced one-dimensionality of the crystallographic structure [3]: $\mathrm{CuO}_{2}$ chains along the $c$ axis with antiferromagnetic interactions between $\mathrm{Cu}$ spins are separated from each other by $\mathrm{GeO}_{2}$ chains.

Below a spin-Peierls transition temperature $T_{\mathrm{SP}}=14 \mathrm{~K}$ the magnetic chains are dimerized which leads to the formation of the non-magnetic ground state separated from the excited triplet states by an energy gap $\Delta \approx 2 \mathrm{meV} \approx 23 \mathrm{~K}$ [4].

In contrast to the previously known organic SP systems, $\mathrm{CuGeO}_{3}$ can easily be doped on both the $\mathrm{Ge}$ and the $\mathrm{Cu}$ sites, offering a possibility of investigating the influence of doping on a SP ground state. The presence of impurities diminishes the temperature of the SP transition and at lower temperatures a Néel ordering 
occurs [5]. The SP dimerization and the antiferromagnetism were found to coexist in doped crystals. This coexistence is suggested to be induced by the disorder in the spin chain resulting from the chemical substitution [6].

Inspired by the measurements performed on doped $\mathrm{CuGeO}_{3}$ crystals, we directed our attention to the other possibilities of introducing disorder. In this paper we present a detailed EPR study on the neutron irradiated $\mathrm{CuGeO}_{3}$ single crystals.

\section{Experimental}

A single crystal of $\mathrm{CuGeO}_{3}$, used in the experiment, was grown from a $\mathrm{CuO}$ flux using a method described in Ref. [7]. It was cut into three pieces. Two of them were exposed to the $0.5 \mathrm{MeV}$ neutron irradiation with fluences of $3.77 \times 10^{17} \mathrm{n} / \mathrm{cm}^{2}$ (sample $S_{1}$, hereafter) and $7.2 \times 10^{17} \mathrm{n} / \mathrm{cm}^{2}\left(\right.$ sample $S_{2}$ ). The third non-irradiated piece (sample $S_{0}$ ) was treated as the reference sample.

During neutron irradiation of samples containing copper the radiation damage results from two different sources: (i) displacements of atoms caused by high-energy $\alpha$ and Co particles which result from the capture of thermal neutrons by the copper isotope ${ }^{63} \mathrm{Cu}$ according to the ${ }^{63} \mathrm{Cu}(\mathrm{n}, \alpha){ }^{60} \mathrm{Co}+0.8 \mathrm{MeV}$ reaction; (ii) displacement of atoms caused by fast neutrons. The effects of radiation-induced chemical changes of the elements contained in the samples were evaluated to be negligible.

EPR measurements were performed using a standard X-band spectrometer (Bruker $300 \mathrm{E}$ ) operating at $9.5 \mathrm{GHz}$ with $100 \mathrm{kHz}$ field modulation. EPR spectra were recorded for an external magnetic field applied along each of the three crystallographic directions in the wide temperature range $2-300 \mathrm{~K}$.

\section{Results and discussion}

The resonance field for both irradiated and non-irradiated samples has an almost constant value in spite of changing temperature, while the absorption intensity and line width strongly depend on temperature. The $g$-values obtained at room temperature for the non-irradiated sample are $g_{a}=2.15, g_{b}=2.26$, and $g_{c}=2.06$ in agreement with previously reported values [8]. After neutron irradiation these values were found to diminish and we obtain $g_{a}=2.10, g_{b}=2.20$, and $g_{c}=2.03$. The possible reason for this $g$-factor deviation is the radiation induced increase in lattice constants which leads to the changes of the crystal field acting on $\mathrm{Cu}^{2+}$ ions.

Figure 1 shows the temperature dependences of the derivative peak-to-peak line width $\Delta H_{p p}^{c}$ obtained for an external field $H$ applied along the $c$ axis for $\mathrm{S}_{0}$, $\mathrm{S}_{1}$, and $\mathrm{S}_{2}$ samples. As can be seen in this figure, after neutron irradiation the line width increases with increasing irradiation dose and tends to saturate above approximately $100 \mathrm{~K}$.

The influence of neutron irradiation on the temperature dependence of the EPR signal intensity of $\mathrm{CuGeO}_{3}$ single crystal is demonstrated in Fig. 2. It can be seen that for the virgin sample $S_{0}$ the intensity has a broad peak around $50 \mathrm{~K}$. After neutron irradiation a large increase in the intensity is observed at low temperatures which depends on the irradiation dose. Above $T_{\mathrm{SP}}=14 \mathrm{~K}$ the difference 


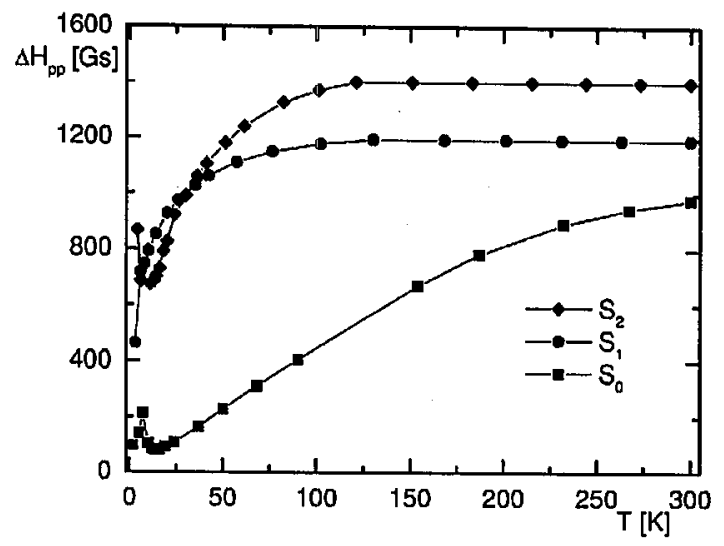

Fig. 1. Temperature dependences of the EPR line width for $\mathrm{CuGeO}_{3}$ before and after neutron irradiation.

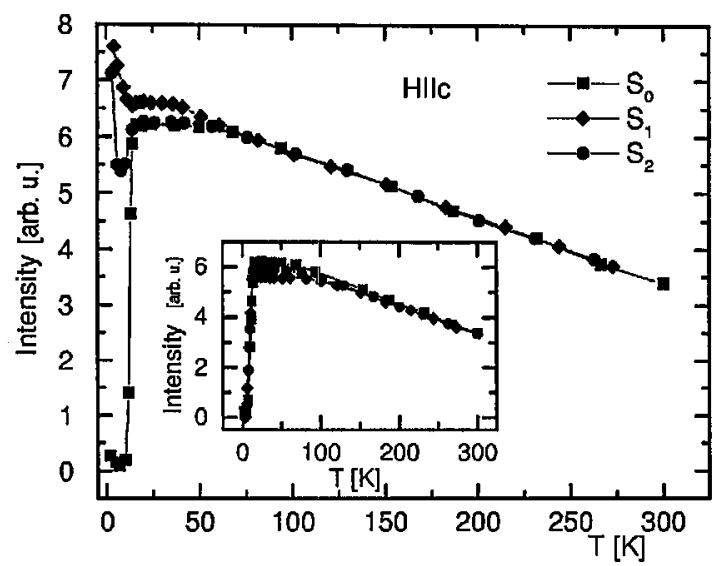

Fig. 2. Temperature dependences of the EPR signal intensity for $\mathrm{CuGeO}_{3}$ before and after neutron irradiation.

between the EPR signal intensity before and after irradiation decreases and for $T>100 \mathrm{~K}$ is within the error limits. The above-mentioned increase in the EPR line intensity has a Curie-like character and may be attributed to a contribution from unpaired $\mathrm{Cu}^{2+}$ ions made free due to neutron irradiation and not participating in the dimerized state. The data in the inset of Fig. 2 show temperature dependences of the EPR signal intensities after the Curie-Weiss terms were subtracted. The drop of the intensity marks the temperature of the SP transition both for non-irradiated and irradiated crystals. The transition temperatures are $T_{\mathrm{SP}}^{0}=14.5 \mathrm{~K}, T_{\mathrm{SP}}^{1}=13.5 \mathrm{~K}$, and $T_{\mathrm{SP}}^{2}=12.5 \mathrm{~K}$ for the $\mathrm{S}_{0}, \mathrm{~S}_{1}$, and $\mathrm{S}_{2}$ samples, respectively. Below the SP transition temperature the EPR signal intensity decreases rapidly with decreasing temperature. This shows that the signals below $T_{\mathrm{SP}}$ come from some thermally activated free spins because most of the spins form a singlet ground state. In this region, the integrated absorption intensity should be proportional to $\exp \left(-\Delta / k_{\mathrm{B}} T\right)$. In order to estimate the value of $\Delta$, we fitted the temperature dependence of EPR signal intensity to $\exp \left(-\Delta / k_{\mathrm{B}} T\right)$ 
using the experimental data below $T_{\mathrm{SP}}$ presented in the inset of Fig. 2. We obtain $\Delta_{0} / k_{\mathrm{B}}=23 \pm 2 \mathrm{~K}, \Delta_{1} / k_{\mathrm{B}}=20 \pm 2 \mathrm{~K}$, and $\Delta_{2} / k_{\mathrm{B}}=18 \pm 2 \mathrm{~K}$ for the $\mathrm{S}_{0}, \mathrm{~S}_{1}$, and $S_{2}$ samples, respectively. The experimentally observed percentage reduction in the energy gap is thus $13 \%$ for the $\mathrm{S}_{1}$ sample and $22 \%$ for the $\mathrm{S}_{2}$ sample while the percentage reduction in the SP transition temperature is $7 \%$ for the $S_{1}$ sample and $14 \%$ for the $\mathrm{S}_{2}$ sample. The above results thus show that neutron irradiation reduces the magnetic energy gap in $\mathrm{CuGeO}_{3}$ much more than it reduces the SP transition temperature. The reduction of the spin gap after neutron irradiation may be related to the radiation induced increase in the lattice constants having effect on the exchange interactions.

Preliminary EPR measurements were also performed on a $\mathrm{CuGeO}_{3}$ single crystal irradiated with higher irradiation dose $1.22 \times 10^{18} \mathrm{n} / \mathrm{cm}^{2}$. For this sample the intensity of the main EPR signal decreased drastically below $4 \mathrm{~K}$ and additional lines with higher resonance fields were observed. It may be attributed to an antiferromagnetically ordered phase formed at lower temperatures which coexists with the SP phase.

\section{Conclusions}

Our results indicate that the main effect of neutron irradiation is to introduce disorder which leads to a partial suppression of both the energy gap and the dimerization within the chains. The results also point towards the possibility of the existence of gapless spin-Peierls state at appropriate irradiation doses. The measurements are still continuing and more detailed results will appear in the forthcoming paper.

\section{Acknowledgments}

This work was supported in part by the Committee for Scientific Research (Poland) under contract No. 2 P03B 13615.

\section{References}

[1] M. Hase, I. Terasaki, K. Uchinokura, Phys. Rev. Lett. 70, 3651 (1993).

[2] M. Hase, I. Terasaki, K. Uchinokura, M. Tokunaga, N. Miura, H. Obara, Phys. Rev. B 48, 9616 (1993).

[3] H. Völlenkle, A. Wittmann, H. Nowotny, Monatsch. Chem. 98, 1352 (1967).

[4] M. Nishi, O. Fujita, J. Akimitsu, Phys. Rev. Lett. 50, 6508 (1994).

[5] L.P. Regnault, J.P. Renard, G. Dhalenne, A. Revcolevschi, Europhys. Lett. 32, 579 (1995).

[6] M. Fabrizo, R. Mélin, Phys. Rev. B 56, 5996 (1997).

[7] M.D. Lumdsen, B.D. Gaulin, H. Dabkowska, Phys. Rev. B 57, 14097 (1998).

[8] G.A. Petrakovskii, K.A. Sablina, A. Vorotynov, A.I. Kruglik, A.G. Klimenko, A.D. Balayev, S.S. Apelsnin, Sov. Phys.-JETP 71, 772 (1990). 